\title{
Plastic Stress and Acoustic Emission as Predictors of Fatigue Life
}

Objectives of this investigation were: first, to utilize a computerized statistical-analysis program to determine relative importance of several parameters as predictors of fatigue life of annealed AISI E4340 steel; secondly, to attempt to relate the phenomenon of acoustic emission to the fatigue characteristics of the steel

\author{
by L. D. Mitchell and J. R. Frederick
}

\begin{abstract}
The high rate of acoustic emission from AISI E4340 annealed steel when it is stressed can be used to accurately indicate the yield stress. Predictive equations for the fatigue life of AISI E4340 annealed steel have been developed by the use of "Multiple Classification Analysis" and "Stepwise Regression" computer programs based on an experimental study of 175 specimens. The acoustic emission from the fatigue specimens was investigated prior to fatiguing, but no parameter of the emission was found to correlate significantly with fatigue life. The high-rate emission which occurs at yield is found to be useful in determining the value of the yield stress. The value of the stress into the plastic range determined in this manner was defined by both statistical approaches as the best predictor of fatigue life. The fatigue stress level was rejected as a lesser predictor. Surface finish in the range of 90 to $350 \mu \mathrm{in}$. was found to have little correlation with fatigue life of this material.
\end{abstract}

\section{Introduction}

The objectives of the investigation reported here were twofold. One was to utilize a computerized statistical-analysis program called "Multiple Classification Analysis" (MCA)' to determine the relative importance of several parameters as predictors of the fatigue life of annealed AISI E4340 steel;

L. D. Mitchell is Senior Research Engineer, E. T. DuPont de Nemours \& Co. Wilmington, Del.; was formerly associated with Dept. of Mechanical Engineering, University of Michigan, Ann Arbor, Mich. J. R. Frederick Engineering, University of Michigan, Ann Arbor, Mich. J. R. Frederick
is Associate Professor, Dept. of Mechanical Engineering, University of Michigan.

Paper was presented at 1970 SESA Spring Meeting held in Huntsville, Ala., on May 19-22. the other was to attempt to relate a phenomenon called acoustic emission to the fatigue characteristics of the steel.

The principal variables studied were the relative amounts of acoustic emission from the test specimen, the amount of stress into the plastic range, $S_{p}$ (as determined from acoustic-emission data) and the applied-stress level. A list of the other parameters, not necessarily independent, that were investigated is shown in Table 1.

TABLE 1-PARAMETERS THAT WERE STATISTICALLY ANALYZED FOR THEIR EFFECT ON FATIGUE LIFE

1. Stress at which a specimen was fatigued, $S$

2. Stress increment in the plastic range as determined acoustically, $\mathrm{S}_{p}$

3. Acoustic emission during the application of a load, $\Sigma E_{L}$

4. Acoustic emission occuring in the vicinity of the yield stress, $\Sigma \mathrm{E}_{H R}$

5. Acoustic emission during the removal of the load, $\Sigma \mathrm{E}_{U}$

6. Lack of concentricity of the gage length with the specimen axis, TIR

7. Surface finish, RMS

8. Temperature, $T$

9. Humidity, $\mathrm{H}$

10. Position of each specimen in the bar from which it came, $P$

11. Bar number, B 


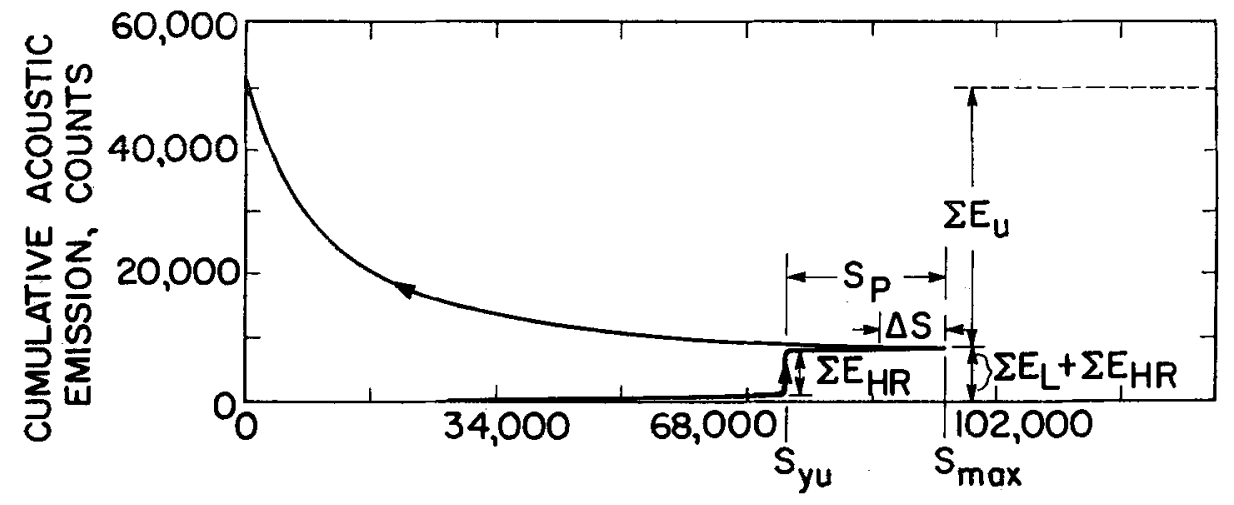

Fig. 1-Schematic representation of the cumulative acoustic emission from the E4340 steel as a function of applied stress. Some of the notation is described in Table 1. Additionally, $\Sigma \mathrm{E}$ is the cumulative emission and $\Delta S$ is the brief delay at the beginning of the unload part of the test during which there is no emission

STRESS, psi

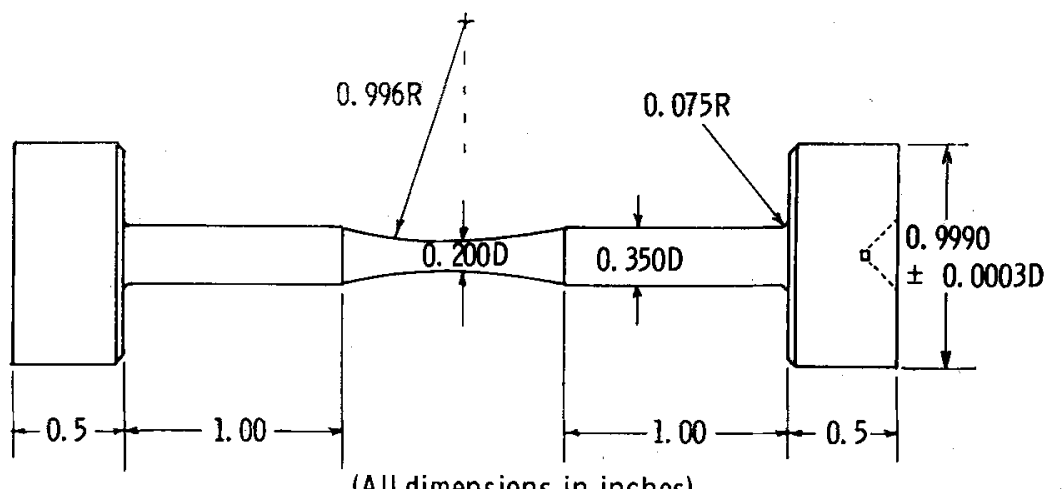

Fig. 2-Test-specimen dimensions

Acoustic emission consists of detectable bursts of low-level elastic waves excited within the test specimen. ${ }^{2}$ It may occur during the application or removal of a stress in either the elastic or the plastic-strain ranges of the specimen. Figure 1 shows schematically how the cumulative emission varies as a load is applied to an annealed E4340 steel specimen and then removed.

TABLE 2-MECHANICAL PROPERTIES OF THE HEAT OF E4340 STEEL USED IN THE TESTS

(Average of Seven Specimens)

\begin{tabular}{lll} 
& $\begin{array}{c}\text { Average } \\
\text { value }\end{array}$ & $\begin{array}{c}\text { Standard } \\
\text { Deviation }\end{array}$ \\
\hline Tensile strength & $111.0 \mathrm{ksi}$ & $3.4 \mathrm{ksi}$ \\
Upper yield stress & $73.0 \mathrm{ksi}$ & $1.7 \mathrm{ksi}$ \\
Strain at fracture, & & \\
$\quad$ 2-in. gage length & $22.4 \%$ & $1.0 \%$ \\
Reduction of area & & \\
$\quad$ at fracture & $47.2 \%$ & $2.8 \%$ \\
Hardness, $R_{c}$ & 22 & 1.1
\end{tabular}

Acoustic emission was selected as a parameter because three of the factors that are known to cause it, namely, dislocation motion, crack nucleation and crack propagation, are closely related to fatigue characteristics. Other mechanisms, inoperative in this material under the test conditions, can cause acoustic emissions that are not necessarily associated with fatigue. They are twinning, grain-boundary sliding and phase change.

\section{Description of the Experiment}

Eight bars of AISI E4340 steel, 1\% $/ 8$ in. in diameter and $12-\mathrm{ft}$ long, from the same heat of material were used to make 175 specimens for this study. Table 2 gives their average measured mechanical properties. The specimen size and shape are shown in Fig. 2.

Acoustic-emission data were obtained on each fatigue specimen prior to the fatigue tests. The acoustic emission was detected by placing a contact microphone in the form of a sensitive piezoelectric transducer on the end of the specimen while 
it was being stressed. The signals thus obtained were amplified and recorded by the use of conventional electronic equipment. Each specimen was stressed to the same level as it was to be stressed in the fatigue tests.

The sensitivity of the system depends on the properties of the transducer and on the acoustical and electrical background noise level. By the use of a specially designed low-noise-level tensiletesting machine and a quiet environment, it was possible to obtain a noise level of $4.0 \mu \mathrm{v}$ at the input of the detection system. A frequency pass-band extending from 300 to $180,000 \mathrm{~Hz}$ was used. The minimum detectable signal under these conditions was calculated to be $0.4 \mathrm{n} / \mathrm{m}^{2}$.

The parameters used in this study that were determined in the acoustic tests were the load emission, the unload emission, the high-rate emission and the value of stress that corresponds to the yield point. The latter was determined by noting the stress at which the high-rate emission starts. This has previously been shown to correspond to the upper yield point while the duration corresponds to the time that the material is going through the upper-lower yield-point extension. ${ }^{3,4}$

The fatigue specimens were stressed axially in tension from zero to their maximum stress level, $S_{\text {max }}$, at a rate of $30 \mathrm{~Hz}$ on a load-controlled hydraulic type of fatigue machine.

\section{Statistical Analysis of Data}

In a statistical analysis, one of the important points is the design of the experiment. Here, a completely randomized design was selected. This provided for a randomization of machining, acoustic testing, and fatigue testing relative to themselves and relative to time. Additionally, the bar number, position number and stress level were randomized relative to all the above variables. In this way, the overall error term is increased but there is little chance of the obliteration of desired correlations.

Two statistical computer programs were used to analyze the experimental data. These programs are designated as (1) Multiple Classification Analysis (MCA) and (2) Stepwise Regression. The MCA program ${ }^{1}$ was used to investigate the effect of each of the several variables on a dependent variable, that is, the total number of cycles to failure $N$. This program does not require independent predictors, a library of functions to be used in the analysis, nor a set of orthogonal data. This provides a capability not available in the regression-analysis approach.

Two distinct models exist for each form of the dependent variable. The first is an additive model described mathematically in eq (1).

$$
\begin{aligned}
N_{i j k l} \ldots= & \bar{N}+\epsilon \Sigma E_{L_{i}}+\epsilon S_{p_{i}}+ \\
& \epsilon T I R_{k}+\epsilon R M S_{1}+\ldots+e_{i j k l} \ldots
\end{aligned}
$$

where $\bar{N}$ is the grand mean for all values of dependent variables; typically, $\epsilon \Sigma E_{i}$ is the effect that the $i$ th category of the acoustic emission has on specimen fatigue life; and $e_{i j k l} \ldots$ is an error term containing that portion of the life not predicted by the ensemble of the predictor variables.

The second model, a multiplicative model, transforms the dependent variable $N$ to $\log _{10} N$. This model in MCA terms is shown in eq (2).

$$
\begin{aligned}
\log _{10} N_{i j k l} \ldots= & \overline{\log _{10} N}+l_{\epsilon} \Sigma_{L_{i}}+l_{\epsilon} S_{p_{i}}+l_{\epsilon} T I R_{k} \\
& +l_{\epsilon} R M S_{1}+\ldots+l_{i j k l} \ldots
\end{aligned}
$$

where $\overline{\log _{10} N}$ is the grand mean for all logarithmic values of $N ; l \epsilon \Sigma E_{L_{i}}$ is the effect of the $i$ th category of the load emission characteristic on the logarithmic life of the specimen, etc., and $l e_{i j k} l \ldots$ is the logarithmic error term containing that portion of the life not predicted by the ensemble of the variables.

The data for each of the predictor variables were inserted into the MCA program by dividing them into an appropriate number of classes, usually from 5 to 10 . For example, the root-mean-square (RMS) surface finish in microinches, which varied from 90 to $350-\mu \mathrm{in}$. RMS, was broken down into five classes and the applied stress into seven classes to cover the range of 80 to $105 \mathrm{ksi}$.

In a real experiment, it is generally impossible to investigate a single dependent variable while holding all but one independent variable constant. Therefore, to gain insight into the actual effects of each variable, MCA-adjusted deviation techniques were used to examine the true dependent-predictor variable relationship. This program generated a set of adjusted deviations that represented the effect each class of the predictor variable had on the dependent variable while all other independent variables were held at a constant level. In the case at hand, all the predictor variables are not necessarily independent. This extends the time for computational convergence. The objective was to generate a predictive equation of the form

$$
\begin{aligned}
Y=b_{o}+b_{1} X_{1}+b_{2} X_{2}+\ldots+ & b_{i} X_{i}+ \\
& \ldots+b_{p} X_{p}
\end{aligned}
$$

where $b_{o}$ is a constant and the $b_{i}$ 's for $i=1,2$, $\ldots, p$ are the coefficients of the individual predictor variables $X_{i}$ which, in turn, can be any specified function or certain interactions of the variables $S, \Sigma E_{L}, \Sigma E_{U}, \Sigma E_{H R}, T I R, R M S, T, H$ and $S_{p}$.

The MCA program evaluates the effect of a class 
TABLE 3-PARTIAL $\beta$ COEFFICIENTS DETERMINED BY THE MULTIPLE-CLASSIFICATION-ANALYSIS PROGRAM

$\begin{array}{ll}S_{p} & 0.860 \\ P & 0.190 \\ T & 0.110 \\ B & 0.064 \\ \Sigma E_{H R} & 0.062 \\ T I R & 0.049 \\ \Sigma E_{U} & 0.038 \\ \Sigma E_{L} & 0.033 \\ R M S & 0.032 \\ H & 0.020\end{array}$

of a predictor variable but it does not generate the mathematical relationship that exists between predictor and dependent variable. With the knowledge obtained from the MCA program, a stepwise-regression analysis was used to develop the mathematical relationships required to predict the fatigue life. The library of functions used for prediction of fatigue life included the predictor variables, functions of any predictor variable, and interactions of predictor variables.

\section{Results of the MCA Program}

Preliminary results of MCA runs indicated that the fatigue stress level, $S$, and the stress in the plastic range, $S_{p}$, were highly dependent and that $S_{p}$ was a better predictor than $S$. Since large intercorrelations such as those found between $S$ and $S_{p}$ hamper convergence in the MCA program, one variable, $S$, was dropped from the program.

The multiplicative model [eq (2)] consistently gave larger multiple correlation coefficients than the additive model [eq (1)] and, hence, appeared to be more applicable to the data. The multiplecorrelation coefficients for the multiplicative model ranged from 0.623 to 0.808 , whereas in the additive model they varied from 0.012 to 0.132 .

The values of the partial $\beta$ coefficients for the predictor variables are shown in Table 3 . The partial $\beta$ coefficients represent that proportion of the variance in the dependent variable that is explained by a particular independent variable while holding all other independent variables constant. It can be seen that the plastic stress as determined from the acoustically determined yield point, $S_{p}$, is the best predictor and all other predictors, including surface finish, are of secondary importance.

\section{Results of the Stepwise-regression Program}

The use of the additive model, [eq (1)] in the stepwise-regression program, resulted in a set of equations which predicted fatigue life rather poorly.
An example of one of these regression equations is

$$
N=532,259+0.533 S^{-2}
$$

where $N$ is the life in cycles and $S$ is the applied stress in units of $10^{4}$ psi. Its coefficient of determination is only 0.162 . However, the use of a multiplicative model in the stepwise-regression program resulted in several different equations for $\log _{10} N$ containing terms involving $S, S_{p}$ and $T$, all of which had much higher values of the coefficient of determination than the additive model. Two of these regression equations whose coefficients of determination are 0.700 and 0.722 , respectively, are:

$$
\log _{10} N=5.8488-0.4947 S_{p}
$$

and

$$
\begin{gathered}
\log _{10} N=50.5-1.002 \times 10^{6} S^{-3}-1.739 \times \\
10^{5} T^{-2}-2.89 \times 10^{-5} T^{3}-\frac{2.80 \times 10^{-6} S^{3}}{1.16 \log S_{p}}
\end{gathered}
$$

where $S$ and $S_{p}$ are in units of $10^{4}$ psi, and $T$ is in units of ${ }^{\circ} \mathrm{F}$.

Other relationships were also derived by the program, but they generally had lower coefficients of determination.

\section{Discussion of Results}

The MCA statistical approach revealed that surface finish within the range of 90 to $350 \mu \mathrm{in}$. RMS for this material has little correlation with fatigue life. In contrast to this result, it is common practice in textbooks on fatigue to attribute the so-called "surface effect" in fatigue to the form of the finishes generated by different machining processes. The low correlation found here would indicate either that for the annealed material used in this work very large surface-finish differences are necessary to affect fatigue life, or that it is not the physical shape of the surface that is important but mainly the damage produced during the different machining processes.

The regression analysis provides the standard error of the slope in the predictive equation and the standard error of $\log _{10} N$ about the fitted regression line. These statistics and others were used to calculate the 95-percent confidence interval for the average value of $\log _{10} N$ for a given value of $S_{p}$ and the 95-percent prediction interval for a future observation of $\log _{10} N$. These calculated intervals are shown in Fig. 3. It was found that five out of all of the data points that were obtained actually fell outside of the 95-percent prediction interval. The expected value of the number of data points outside of the predictive interval is 7.25 specimens.

The regression analysis consistently rejected $S$ and used the acoustically determined value of the 
Fig. 3-Ninety-five percent interval for the average value of $\log _{10} N$ and the 95 percent prediction interval for a future observation of $\log _{10} N$ annealed $E 4340$ steel; $\log _{10} N=4.95$

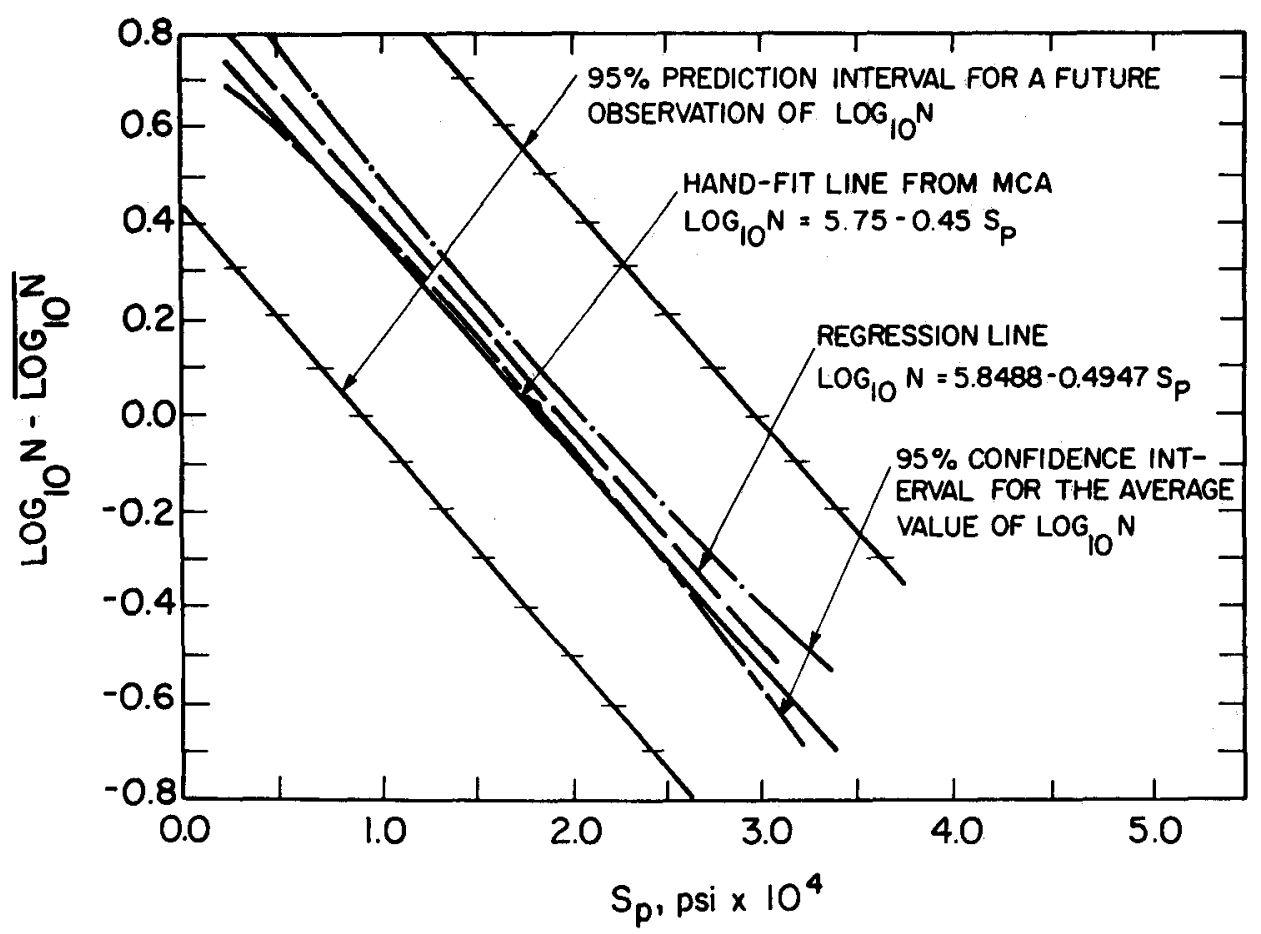

plastic stress, $S_{p}$, as the best predictor. When $S$ was forced into the program to give an equation between $\log _{10} N$ and $S$, a coefficient of determination 4.5 percent lower than that for $S_{p}$ was obtained. This result is in agreement with the observation of Tavernelli and Coffin ${ }^{5}$ that a direct measurement of plastic deformation under typical applied fatigue stresses offers a better prediction of fatigue life.

It is of interest to observe that predictive eq (4) indicates a fatigue life close to $10^{6}$ cycles when the specimen is stressed at the upper-lower yield point. This is in agreement with published data on AISI E4340 which show that the endurance limit occurs at or after $10^{6}$ cycles. ${ }^{6}$

The low values of the partial $\beta$ coefficients obtained for the acoustic-emission data indicate that there are insufficient damage-related events occurring in the first cycle of a fatigue test to produce enough acoustic emission for prediction purposes. There is evidence from work now in progress at this laboratory that the acoustic emission as measured continuously throughout the fatigue test is greater for short-lived specimens than from those specimens having longer lives and that this difference is evident in some specimens at a small percentage of the fatigue life.

\section{Conclusions}

Both the MCA and regression programs show that the acoustically determined plastic stress, $S_{p}$, investigated in this work is more highly correlated with the finite fatigue life of AISI E4340 annealed steel than the more commonly used value of the total applied stress, $S$. Thus, fatigue life of E4340 in the annealed condition is intimately related to the plastic stress applied to the material. The other acoustic-emission characteristics do not measure this plastic behavior. Moreover, it is concluded that the fatigue limit $\left(10^{6}\right.$ cycles $)$ in tension-tension (zero to maximum load) fatigue is at the upper-lower yield point in annealed AISI E4340 steel.

\section{Acknowledgment}

This work was supported in part by the National Science Foundation.

\section{References}

1. Andrews, F., Morgan, J. and Sonquist, J. A., "Multiple Classification Analysis," The Institute for Social Research, The University of Michigan, Ann Arbor, Mich. (May 1967).

2. Fisher, R. M. and Lally, J. S., "Microplasticity Detected by an Acoustic Technique," Can. Jnl. Phys. 45, 1147-1159 (1967).

3. Borchers, H. and Tensi, H. M., "Eine verbesserte piezoelektrische Methode zur Untersuchung von Vorgängen in Metallen bei mechanischer Beanspruchung und bei Phasenanderung," Z. Metall., 51 (4), 212-218 (1960).

4. Borchers, H. and Tensi, H. M., "Piezoelektrische Impulsmessungen während der mechanischen Beanspruchung von Al Mg 3 und Al 99," Z. Metall., 53 (10), 692-695 (1962).

5. Tavernelli, J. F and Coffin, L. F., Jr., A Compilation and Interpretation of Cyclic Strain Fatigue Tests on Metals, Trans. ASM, 51, 438 (1959).

6. Grover, H. J., Gordon, S. A. and Jackson, L. R., Fatigue of Metals and

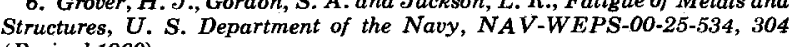
(Revised 1,960) 